\title{
INVESTIGACIONES
}

\section{Valor del conocimiento pedagógico para la docencia en Educación Secundaria: diseño y validación de un cuestionario*}

\author{
The value of pedagogical knowledge for teaching Secondary School: \\ design and validation of a questionnaire
}

\author{
Ana Martin-Romera, ${ }^{a}$ Enriqueta Molina Ruiz ${ }^{b}$ \\ aBecaria FPU del Departamento de Didáctica y Organización Escolar, Universidad de Granada \\ Telf.: (34) 670274185. Correo electrónico: amromera@ugr.es \\ bDepartamento de Didáctica y Organización Escolar, Universidad de Granada \\ Correo electrónico: emolina@ugr.es
}

\section{RESUMEN}

Los docentes y su formación son determinantes en el aprendizaje y la mejora de la calidad de la educación. Reclutar a los mejores docentes requiere de buenas políticas de formación inicial que asuman el reto de atender a las demandas y necesidades de los profesores en la práctica. Este trabajo presenta el proceso de construcción y validación de un cuestionario que permite recoger el valor que el profesorado de enseñanza secundaria concede al conocimiento pedagógico en su actuación profesional y a la necesidad de formación pedagógica. En este trabajo se muestra el procedimiento desarrollado para estimar la validez de contenido y consistencia interna del cuestionario. El proceso de construcción y validación derivó en un cuestionario constituido por tres secciones dirigidas a recoger la valoración del Conocimiento Pedagógico y Competencias docentes para el ejercicio de la profesión, y los Elementos deseables en la formación inicial pedagógica.

Palabras clave: formación de profesores, Educación básica, instrumentos de evaluación, validez, estudio piloto.

\section{ABSTRACT}

Teachers and their training are determining factors in learning and improving the quality of education. Recruiting the best teachers requires good initial training policies that take up the challenge of meeting the demands and needs of teachers in their practice. The study describes the construction and validation process of a questionnaire designed to discover the value that high school teachers give to pedagogical knowledge in their professional performance and the need for pedagogical training. In this paper the procedure developed to estimate the questionnaire content validity and its internal consistency are presented. The construction and validation process resulted in a questionnaire consisting of three sections aimed at collecting the valuation of Pedagogical Knowledge and Teaching Competences for the exercise of the profession, and the Desirable Elements on preservice pedagogical training.

Key words: teacher education, basic education, evaluation instruments, validity, pilot study.

Esta investigación forma parte de un proyecto de tesis doctoral financiado mediante una beca predoctoral del programa de ayudas para la Formación de Profesorado Universitario del Ministerio de Educación, Cultura y Deporte (España). 


\section{INTRODUCCIÓN}

Los cambios sociales y culturales experimentados por la Educación Secundaria en las últimas décadas, y concretamente la extensión de la obligatoriedad de la Educación Primaria y Secundaria a toda la población, han supuesto transformaciones sustanciales en la profesionalidad de los docentes (Imbernón, 2014).

Ser profesor de Secundaria en la actualidad requiere algo más que dominar conceptualmente una materia (Hernández, 1998). Numerosos son los autores que se pronuncian en ese sentido indicando que además del conocimiento de la disciplina, es necesario "saber enseñarlo" dando relevancia también a otros factores tales como conocimiento didáctico, del alumnado, del contexto educativo y de las interacciones que se desarrollan en el mismo (e.g. Darling-Hammond, 2006; Darling-Hammond \& BaratzSnowden, 2005; Shulman, 2005; Vaillant \& Marcelo, 2015). Para ello, se hace necesario un conocimiento pedagógico especializado sobre las características de los estudiantes y de la adolescencia, la gestión del aula, la metodología didáctica y el trabajo en equipo (Esteve, 2003; Marcelo, 2009; Perrenoud, 2004). Sin embargo, diversas investigaciones (Pontes, Ariza \& Del Rey, 2010) han mostrado que se trata de un conocimiento, por lo general, que es escasamente valorado por el profesorado en el momento de la formación, aunque su importancia parece reconocerse en el desempeño de la profesión a la par que se constatan necesidades formativas en tal sentido (Terigi, 2011). Al respecto, diversos estudios (e.g. Ferrández-Berrueco \& Sánchez-Tarazaga, 2014; Sánchez-Tarazaga, 2012) han mostrado que el profesorado en ejercicio, aunque manifiesta una mayor valoración hacia las competencias docentes asociadas al dominio de la disciplina, valora de forma positiva otras competencias docentes, de carácter pedagógico, que se perfilan como necesarias en la actualidad.

A pesar de que se ha asignado a este momento formativo la función de dotar a los futuros docentes del conocimiento pedagógico base (Imbernón, 2007) que les permita seguir aprendiendo a lo largo de su trayectoria profesional (Bransford, Darling-Hammond \& LePage, 2005), el sistema de formación inicial y ,especialmente, la forma de estructurar los programas de formación inicial del profesorado de Secundaria -desde modelos profesionales consecutivos- son destacadas, en la literatura especializada, como posibles causas de la valoración manifestada por el profesorado hacia dicho conocimiento (e.g. Esteve, 2003; Terigi, 2011).

La formación inicial del profesorado de Secundaria en el contexto español no siempre se ha concebido del mismo modo, como ha ocurrido con la labor de este profesional. En su origen, la formación inicial constituía una formación disciplinar que priorizaba el conocimiento y la erudición científica del docente. Con la Ley General de Educación (1970), se establece un sistema reglado de formación inicial de carácter pedagógico y didáctico. Dicho sistema, que se ha venido desarrollando durante casi cuatro décadas, exigía al profesorado para acceder a la docencia una formación pedagógica mínima (e.g. Curso de Aptitud Pedagógica) una vez finalizados los estudios de licenciatura. Recientemente, con la llegada del proceso de convergencia europeo, -a partir del curso académico 2009-2010 comienza a implantarse un nuevo plan de formación inicial de carácter pedagógico a nivel de posgrado: "Máster en Formación del Profesorado de Educación Secundaria".

A pesar de la reciente reforma, son numerosas las voces que siguen cuestionando el potencial de la formación inicial para proporcionar al futuro profesorado la profesionalidad demandada (e.g. Bolívar \& Bolívar Ruano, 2012; Marcelo, 2009). El profesorado valora 
escasamente la formación inicial pedagógica y la califica como "insuficiente", "en exceso teórica", o "poco realista" (Bolívar, 2006; Tardif, 2004). Por otro lado, desde la academia, son numerosos los trabajos que han analizado el nuevo plan formativo y, con prospectiva, han destacado posibles áreas de mejora del actual plan formativo atendiendo de forma específica a algunos de sus componentes como la estructura curricular (e.g. Bolívar y Bolívar Ruano, 2012; Escudero, 2009), las competencias de la titulación (e.g. Sarramona, 2012) o el sistema de prácticas docentes (e.g. González Sanmamed, 2009). En concordancia con el profesorado, dichos trabajos orientan su discurso hacia la necesidad de una formación pedagógica que responda a la especificidad y las circunstancias que rodean al trabajo docente en esta etapa educativa (Escudero, 2009; González Sanmamed, 2009; Rodríguez et al., 2016). Afrontar este reto, tal y como destacan los trabajos de Bolívar y colaboradores (e.g. Bolívar y Bolívar Ruano, 2012; Valdés, Bolívar \& Moreno, 2015), requiere que las instituciones de Educación Superior asuman el reto y determinen cuáles han de ser los propósitos de la formación (para qué), qué necesitan los profesores para afrontar las demandas profesionales derivadas de su práctica (qué), plantear diseños adecuados que atiendan a la formación demandada, así como políticas que los apoyen (cómo).

En un intento de contribuir a tal cometido, y como parte de una investigación más amplia dirigida a contribuir a la mejora de la formación inicial pedagógica del profesorado de Enseñanza Secundaria en el contexto español (Martin-Romera, Molina Ruiz \& Olmo, 2015), abordamos en esta aportación el proceso de construcción y validación de un cuestionario que nos permitirá conocer el valor que el profesorado de enseñanza secundaria concede al conocimiento pedagógico en su preparación y actuación profesional, así como sus necesidades de formación en tal sentido.

\section{MÉTODO}

Presentamos los elementos integrantes del diseño y validación del cuestionario, enmarcados en una metodología de investigación de corte descriptivo e interpretativo dirigida a informar sobre las propiedades psicométricas del cuestionario. Por su relevancia metodológica y profundidad este trabajo atiende a las evidencias de validez de contenido y consistencia interna del cuestionario. Con la finalidad de ilustrar el contenido del cuestionario valorado y aplicado, en un primer momento describimos las tareas que fueron emprendidas para el diseño del cuestionario; posteriormente, en un segundo apartado describimos el proceso de validación desarrollado.

\subsection{ELABORACIÓN DEL CUESTIONARIO: DEFINICIÓN DE LOS ASPECTOS GENERALES Y DISEÑO}

El primer paso para la elaboración del cuestionario quedó establecido por la propia naturaleza de los objetivos de la investigación. Identificamos al cuestionario como la herramienta más adecuada para abordar el objeto de estudio, que nos permite acceder a las muestras necesarias para extrapolar los resultados a las poblaciones de interés.

Una segunda tarea fue la delimitación de los objetivos específicos que deseamos conseguir, aspecto que guiaría el proceso de diseño de los cuestionarios mediante la determinación de la información que se precisa para responder a los objetivos de la investigación. 
Una primera fase estuvo centrada en el desarrollo de un conjunto de tareas dirigidas al diseño del cuestionario -generar los temas, decidir las claves de medida y escribir los ítems-. Dichas tareas se enmarcaron en un proceso de operacionalización (Cea D’Ancona, 2001; Solís, 2013 Cit. en Hernández, Fernández \& Baptista, 2014) que consistió en definir y transformar las variables teóricas de la investigación en las variables empíricas, materializadas en las dimensiones e ítems del cuestionario (Figura 1). Este proceso fue especialmente relevante para determinar el método para medir las variables empíricas y la posibilidad de realizar inferencias de los datos a la población.

Figura 1. Proceso de operacionalización.

De los objetivos de investigación al cuestionario

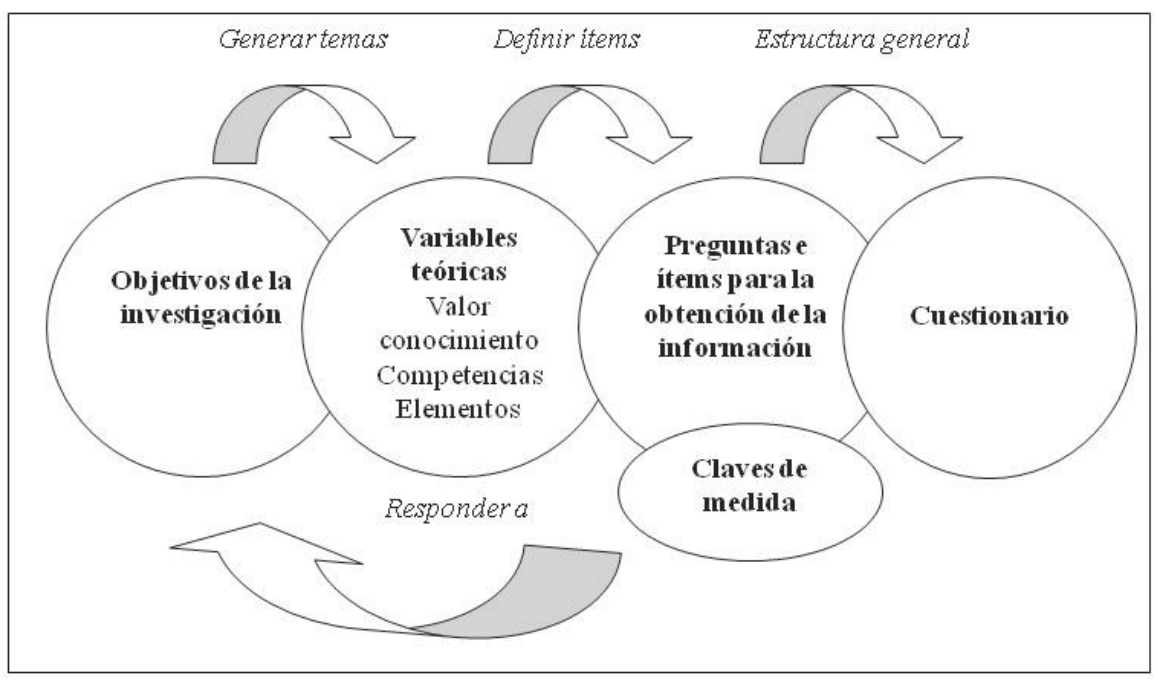

Fuente: elaboración propia.

La primera tarea en el diseño del cuestionario consistió en determinar qué información se requiere para responder a los objetivos de investigación, es decir, convertir los objetivos en la información que se requiere recopilar o, lo que es lo mismo, esbozar los tópicos que se quieren incluir en el cuestionario (Mertens, 1998). Realizada una extensa revisión de la literatura, para llegar a un conocimiento amplio y completo de la temática de estudio, definimos las variables teóricas, dimensiones e indicadores atendiendo a los objetivos de la investigación propuestos. De forma coherente a estos, fueron definidos los ítems y las claves de la medida. El cuestionario quedó estructurado en un conjunto de ítems de tipo sociodemográfico y actitudinal. Para las preguntas sociodemográficas (Datos de identificación del cuestionario) se formularon preguntas de tipo nominal (por ejemplo, Formación recibida, Categoría profesional, Especialidad, etc.) y escalar (Años de experiencia docente). Para las preguntas de tipo actitudinal (dimensiones C.1, C.2 y C.3; Tabla 1), se optó por una medida de tipo Likert de 5 puntos, que permite a los encuestados posicionarse en el lugar que mejor refleja sus creencias u opiniones sobre el enunciado (Cohen, Manion \& Morrison, 2011; McMillan \& Schumacher, 2011). 
Una segunda tarea estuvo dirigida a diseñar y escribir los ítems del cuestionario siguiendo las indicaciones de la literatura (e.g. Cohen et al., 2011; Morales Vallejo, 2010): elaborar ítems claros, cortos y sencillos, evitar ítems con dos objetivos, adaptados a los encuestados, evitar sesgos en la cumplimentación, etc.

Finalmente, procedimos a dar el formato general al instrumento, prestando especial cuidado para su distribución y organización general. La versión inicial del cuestionario quedó estructurada en tres secciones con un total de 150 ítems de formato cerrado y 7 de formato abierto (Tabla 1).

Tabla 1. Correspondencia entre objetivos de la investigación e información requerida

\begin{tabular}{|c|c|}
\hline OBJETIVOS DE INVESTIGACIÓN & DIMENSIONES Y Nº DE ÍTEMS \\
\hline \multicolumn{2}{|c|}{ C.1. Valor del conocimiento pedagógico } \\
\hline $\begin{array}{l}\text { Descubrir si existen coincidencias caracter- } \\
\text { izadoras de las diferentes etapas profesio- } \\
\text { nales en el modo de valorar los profesores } \\
\text { de enseñanza secundaria el conocimiento } \\
\text { pedagógico en el ejercicio de la profesión }\end{array}$ & $\begin{array}{l}\text { A. (1-3): Aspectos necesarios para ser profesor } \\
\text { E. (20-24): Modos de adquirir conocimiento ped- } \\
\text { agógico } \\
\text { F. Características de la formación inicial pedagógica } \\
\text { (p. abierta) }\end{array}$ \\
\hline $\begin{array}{l}\text { Descubrir la posible evolución experimen- } \\
\text { tada por profesores de enseñanza secundar- } \\
\text { ia con larga trayectoria profesional respecto } \\
\text { al reconocimiento de la importancia de po- } \\
\text { seer conocimiento pedagógico para el de- } \\
\text { sarrollo de su docencia actual, describiendo } \\
\text { el proceso y elementos influyentes }\end{array}$ & $\begin{array}{l}\text { B. (4-9): Descubrimiento de la importancia de po- } \\
\text { seer conocimiento pedagógico. } \\
\text { C. (10-16): Factores influyentes en el reconocimien- } \\
\text { to de la importancia del conocimiento pedagógico } \\
\text { D. (17-19): Evolución en la valoración del cono- } \\
\text { cimiento pedagógico } \\
\text { "Otros": } 2 \text { preguntas abiertas }\end{array}$ \\
\hline \multicolumn{2}{|c|}{ C.2. Competencias docentes } \\
\hline $\begin{array}{l}\text { Averiguar las competencias docentes mejor } \\
\text { valoradas por los profesores para el buen } \\
\text { desempeño de sus funciones como en- } \\
\text { señantes }\end{array}$ & $\begin{array}{l}\text { I. (1-3): Realizar la evaluación inicial del proceso } \\
\text { educativo } \\
\text { II. (4-9): Planificar los procesos de enseñanza-apren- } \\
\text { dizaje } \\
\text { III. (10-17): Desarrollar la enseñanza } \\
\text { IV. (18-20): Orientar a alumnos y familias } \\
\text { V. (21-27): Participar en el centro } \\
\text { VI. (28-32): Actualización e implicación en la pro- } \\
\text { fesión } \\
\text { VII. (33-36): Investigar en el aula } \\
\text { VIII. (37-39): Innovar } \\
\text { IX. (40-44): Desarrollo personal y ético de la pro- } \\
\text { fesión } \\
\text { X. (45-51): Evaluar el proceso de enseñanza-apren- } \\
\text { dizaje }\end{array}$ \\
\hline
\end{tabular}




\begin{tabular}{|l|l|}
\hline \multicolumn{2}{|c|}{ C.3. Elementos deseables } \\
\hline & H. (1-10): Principios formativos \\
& I. (11-17): Objetivos \\
& J. (18-25): Saberes \\
Averiguar los elementos considerados esen- & K. (26-29): Fórmulas \\
ciales en programas de formación inicial & L. (30-36): Planificación \\
pedagógica para lograr una adecuada prepa- & M. (37-43): Procesos metodológicos \\
ración en el desarrollo de la actividad do- & N. (44-47): Recursos (espacios, materiales, otros) \\
cente & O. (48-57): Agentes formadores (tipos, requisitos) \\
& P. (58-66): Evaluación \\
& Q. (67-74) Prácticas docentes \\
& "Otros": 4 preguntas abiertas \\
\hline
\end{tabular}

Fuente: elaboración propia.

\subsection{VALIDACIÓN DEL CUESTIONARIO}

Una vez se han definido las dimensiones e ítems de los cuestionarios, el siguiente paso consistió, como aconseja Cea D’Ancona (2001), en comprobar hasta qué punto dicha operacionalización reúne los criterios de calidad antes de ser aplicados a una muestra de las poblaciones de interés. Al respecto, McMillan y Schumacher (2011) aconsejan que son necesarios dos pasos para tener retroalimentación antes de aplicar un cuestionario en un estudio: valoración de su contenido y prueba piloto del cuestionario. Ambos procedimientos fueron aplicados en esta investigación para probar las bondades de validez de contenido y consistencia interna del cuestionario.

La validez de contenido se refiere a un proceso que tiene como objetivo proporcionar evidencias de adecuación del instrumento al área de contenido que se espera medir (Muijs, 2011). La validez de contenido desarrollada se dirigió a obtener indicadores de la relevancia, adecuación y claridad del contenido del cuestionario mediante la evaluación de jueces expertos (Escobar \& Cuervo, 2008). Dicho procedimiento fue abordado desde una estrategia metodológica de integración de dos fuentes de datos y de métodos (Denzin \& Lincoln, 2005) con la finalidad de evaluar la consistencia de la información proporcionada por los jueces. Esta estrategia consistió en solicitar a un grupo de expertos de reconocida experiencia en diversas áreas de interés para la investigación la valoración del contenido y forma del cuestionario. Con la finalidad de triangular información derivada de diversos ámbitos de interés para la investigación recogimos la valoración de personas que poseen un conocimiento profundo de la situación y desarrollan su labor en el campo profesional de la Educación Secundaria, el profesorado.

Una vez estudiadas las bondades del contenido y forma, y acometidas las pertinentes mejoras, procedimos a realizar un estudio piloto de los ítems del cuestionario. La aplicación piloto consiste en aplicar un instrumento a una muestra con la finalidad de comprobar diversas condiciones para su aplicación efectiva: la comprensión de las preguntas e instrucciones, duración del cuestionario, la receptividad de la muestra al instrumento (Cohen et al., 2011) o estimar la fiabilidad del instrumento (McMillan \& Schumacher, 2011). 
En esta investigación procedimos a una administración de la prueba de papel y lápiz de los cuestionarios con la finalidad de analizar el funcionamiento (comprensión y aplicación) de los cuestionarios y estimar la fiabilidad de los mismos mediante dos procedimientos: análisis de ítems y consistencia interna.

\subsubsection{Participantes}

Los métodos de muestreo adoptados respondieron a procedimientos de tipo no probabilístico e intencional.

La selección de los expertos participantes en la validez de contenido se realizó mediante muestreo experto (expert sampling, Singh, 2007). Este procedimiento consiste en seleccionar un grupo de sujetos de acuerdo a criterios que atiendan a la experiencia específica demostrable o reconocida en un campo.

Como hemos comentamos previamente, seleccionamos una muestra de especialistas con experiencia demostrable en diversas áreas de interés para nuestro estudio (investigación, formación docente y contexto en que los profesores de Educación Secundaria desarrollan su acción docente). (Tabla 2).

Tabla 2. Ámbito de experiencia y actuación profesional de los jueces expertos participantes

\begin{tabular}{|l|c|c|c|c|}
\hline \multirow{2}{*}{ ÁMBITO DE EXPERIENCIA } & \multicolumn{3}{c|}{ ÁMBITO PROFESIONAL } & \multirow{2}{*}{ Total } \\
\cline { 2 - 4 } & Universidad & Inspección & Máster FPES & \\
\hline Investigación & 2 & 0 & 1 & 3 \\
\hline Formación docente & 2 & 0 & 1 & 3 \\
\hline Contexto de la profesión docente & 1 & 2 & 1 & 4 \\
\hline Total & 5 & 2 & 3 & 10 \\
\hline
\end{tabular}

Fuente: elaboración propia.

Los expertos participantes fueron seis mujeres y cuatro hombres, cuya experiencia profesional oscilaba entre 11 y 35 años $(X=19,29 ; S D=8,55)$ : i) tres especialistas en formación inicial docente relacionados con el contexto de Educación Superior y la coordinación de especialidad del Máster de Formación de Profesorado de Secundaria de la Universidad de Granada (España); ii) tres profesores universitarios especialistas en la elaboración de pruebas e instrumentos de investigación de la Universidad de Granada; y iii) cuatro profesionales conocedores del contexto -inspectores de educación- y la profesión docente en Educación Secundaria -catedrático de enseñanza secundaria con 13 años de experiencia docente en Educación Secundaria y 25 años en Educación Superior-.

La muestra de profesores participantes en la validación del contenido y forma del cuestionario fue seleccionada de forma intencional para atender a las características de la población objeto de estudio: profesorado de Enseñanza Secundaria de las diversas especialidades, con experiencia docente en diversos contextos y niveles educativos (Tabla 3). 
Tabla 3. Profesorado participante en la validez de contenido

\begin{tabular}{|l|c|c|c|}
\hline \multirow{2}{*}{ ESPECIALIDAD } & \multicolumn{2}{|c|}{ NIVEL EDUCATIVO } & \multirow{2}{*}{ Total } \\
\cline { 2 - 4 } & ESO y/o Bachillerato & Formación Profesional & \\
\hline Ciencias* & 2 & 1 & 3 \\
\hline Educación Física & 1 & 0 & 1 \\
\hline Filosofía & 1 & 0 & 1 \\
\hline Francés & 1 & 0 & 1 \\
\hline Geografía e Historia & 1 & 0 & 1 \\
\hline Inglés & 1 & 0 & 1 \\
\hline Lengua y Literatura & 1 & 0 & 1 \\
\hline Orientación Educativa & 1 & 0 & 1 \\
\hline Total & 9 & 1 & 10 \\
\hline
\end{tabular}

Nota $=*$ No indicó especialidad del cuerpo de profesores.

Fuente: elaboración propia.

La muestra participante estuvo compuesta por diez profesores de enseñanza secundaria de la provincia de Granada (siete hombres y tres mujeres), con experiencia docente -entre los 4 y 33 años $(X=18,10 ; S D=11,37)$ - en diversos niveles de Educación Secundaria (Obligatoria y Postobligatoria) (Tabla 3).

En la aplicación piloto del cuestionario participaron 38 profesores ( 24 hombres y 14 mujeres) que ejercen su labor en la provincia de Granada y diferentes niveles educativos (Tabla 4).

Tabla 4. Profesorado participante en la prueba piloto

\begin{tabular}{|c|c|c|}
\hline Edad & N & Porcentaje de la muestra \\
\hline $25-30$ & 3 & 7,9 \\
\hline $31-36$ & 6 & 15,8 \\
\hline $37-42$ & 6 & 15,8 \\
\hline $43-48$ & 7 & 18,4 \\
\hline $49-54$ & 7 & 18,4 \\
\hline 55 o m & 4 & 10,5 \\
\hline NS/NC & 5 & 13,2 \\
\hline Total & 38 & 100,0 \\
\hline
\end{tabular}




\begin{tabular}{|c|c|c|}
\hline Niveles & $\mathrm{N}$ & Porcentaje de la muestra \\
\hline E.S.O. & 22 & 58,0 \\
\hline E.S.O. y Bachillerato. & 11 & 29,0 \\
\hline E.S.O. y F.P. & 2 & 5,2 \\
\hline F.P. & 1 & 2,6 \\
\hline E.S.O. y Universidad & 1 & 2,6 \\
\hline NS/NC & 1 & 2,6 \\
\hline Total & 38 & 100,0 \\
\hline
\end{tabular}

Nota: NS/NC= No sabe/No contesta; E.S.O.= Educación Secundaria Obliga-

toria; F.P.= Formación Profesional.

Fuente: elaboración propia.

Con una experiencia docente que oscila entre uno y 37 años $(X=17,75 ; S D=9,581)$, en su mayoría, el profesorado ejerce docencia en Educación Secundaria Obligatoria (94,8\% del total), compaginando, en algunos casos, con otras enseñanzas como Formación Profesional (5,2\%), Bachillerato (29\%), o docencia en Educación Superior (2,6\%).

\subsubsection{Instrumentos y medidas}

Para la recogida de información se utilizaron los siguientes instrumentos y medidas:

A la muestra de expertos y profesores participantes en el juicio de expertos se administró un instrumento construido ad $h o c^{1}$, cuyo diseño estuvo inspirado en las indicaciones metodológicas de Escobar \& Cuervo (2008) y el modelo desarrollado por Burgos (2007) (ver Anexo 1).

La plantilla de validación queda estructurada en tres secciones:

- Datos de identificación del participante: actividad laboral actual, años de experiencia y sector profesional.

- Valoración de los ítems del cuestionario. Esta sección solicita a los participantes una valoración cuantitativa de los grupos de ítems, en una escala Likert de cuatro puntos $-1=$ Nada; $2=$ Poco; $3=$ Bastante; 4=Mucho - sobre elementos de la validez de contenido (Sireci \& Faulkner-Bond, 2014): Relevancia para el dominio, Adecuación de los ítems al dominio y Claridad de los ítems.

- Valoración global del cuestionario. Una tercera sección se dirige a recoger las valoraciones de los jueces sobre el grado de adecuación $-1=\mathrm{Nada}$; 2=Poco; $3=$ Bastante; 4=Mucho - del cuestionario a un conjunto de aspectos que han de ser tomados en consideración durante el proceso de construcción de cuestionarios -destacados en trabajos como los desarrollados por McMillan y Schumacher (2011) o Muijs (2011)-, fuertemente asociados a los procesos de elaboración

Una versión inicial del instrumento fue revisada en su contenido por dos investigadores universitarios con experiencia consolidada en investigación educativa y construcción de pruebas. 
(apariencia, claridad y extensión de las instrucciones, formato de los ítems, orden de las preguntas), recogida (tiempo de cumplimentación, respeto al anonimato de los encuestados) o interpretación de datos (información objetiva, libre de sesgos), y que pueden atentar contra la validez y fiabilidad del cuestionario.

A la muestra piloto se le administró el cuestionario objeto de la validación. El instrumento consta de una parte introductoria con una breve descripción de la temática de estudio y las instrucciones para su cumplimentación. Tras la misma, presenta un apartado referido a los datos sociodemográficos de interés para la investigación (Datos de Identificación), recopilados a través de preguntas de tipo nominal y escalar.

Se estructura en tres secciones que se corresponden con las variables teóricas y empíricas de la investigación:

- Valor del conocimiento pedagógico para el ejercicio de la profesión docente, compuesta por 25 ítems positivos e invertidos ${ }^{2}$ estructurados en cinco grupos de ítems que hacen referencia a dos dimensiones: a) Valoración del conocimiento pedagógico: Aspectos necesarios para ser profesor; Descubrimiento de la importancia; Factores influyentes; Evolución en la valoración; y b) Fuentes de adquisición: Modos de adquisición; Características que debería tener la formación inicial para proporcionar conocimiento pedagógico. Los cinco primeros grupos de ítems miden el grado de acuerdo, en una escala Likert de cinco puntos que oscila de $\mathrm{N}=\mathrm{Nada}$ (1) a $\mathrm{M}=$ Mucho (5).

- La segunda sección, Competencias docentes en el ejercicio de la profesión, se compone de 40 ítems estructurados en diez dimensiones cada una de ellas referida a una función docente. La escala está dirigida a medir la importancia concedida por el profesorado en una escala de cinco puntos donde $N$ es Nada importante y $M$ Muy importante.

- La última sección denominada Elementos deseables en la Formación Inicial Pedagógica está compuesta de 39 ítems estructurados en nueve dimensiones referidas a componentes formativos. La sección está dirigida a medir la importancia concedida, de 1 (Nada) a 5 (Mucho), por los profesores al conjunto de componentes.

Una última pregunta, de carácter abierto, se dirige a recoger otras consideraciones sobre la temática.

\subsubsection{Procedimiento de análisis}

Recogidas las valoraciones de los jueces y profesores, el análisis se enfocó a los siguientes procedimientos:

- Análisis de la consistencia inter-jueces. Este análisis estuvo sustentado en los análisis que se exponen a continuación:

Para esta sección fueron definidos tres ítems invertidos y en negativo con dos propósitos, minimizar los posibles sesgos asociados a la aquiescencia, y atenerse a los objetivos de investigación (Véase Tabla I). 
Frecuencia y tendencia central (media y desviación estándar) dirigidos a analizar la homogeneidad en las valoraciones de los jueces expertos y profesores.

Pruebas de contraste U de Mann-Whitney y H de Kruskal-Wallis con la finalidad de obtener evidencias de concordancia en las valoraciones en función del tipo de experto (profesorado o experto) y en función del área de especialización, donde la validez quedó establecida cuando la prueba resultó no significativa atiendo a un nivel de probabilidad del $95 \%(\mathrm{p}>, 05)$.

- $\quad$ Análisis de la adecuación de los ítems del cuestionario.

Una vez calculados los estadísticos de consistencia inter-jueces procedimos a revisar la adecuación de los ítems a los criterios de validez de contenido adoptados, mediante el coeficiente de validación V de Aiken (Aiken, 1985). Este método ofrece una magnitud que informa sobre la proporción de jueces que manifiestan una valoración positiva sobre el objeto valorado, que puede adoptarse como criterio para tomar decisiones en cuanto a la pertinencia de revisar o eliminar los ítems. Esta magnitud fue calculada mediante la siguiente fórmula:

$$
\begin{array}{l|l}
V=\frac{\chi-l}{k} & \begin{array}{l}
\chi=\text { media de calificaciones de los jueces } \\
1=\text { valor de calificación más bajo de la escala } \\
\mathrm{k}=\text { rango de los valores posibles de la escala de valoración }
\end{array}
\end{array}
$$

El resultado ha sido interpretado atendiendo al método score desarrollado por Penfield y Giacobbi (2004) asumiendo un nivel de confianza del 95\% ( $\mathrm{Vp}=, 5)$. Dicho procedimiento nos ha permitido calcular con un nivel de probabilidad la adecuación del contenido de los grupos de ítems atendiendo a cada grupo de jueces participantes ${ }^{3}$. Aunque el coeficiente se ha dirigido a estimar la relevancia del ítem respecto a su constructo, calculamos el coeficiente e intervalos de confianza asociados para los tres criterios de validez de contenido considerados.

Atendiendo a los análisis estadísticos aplicados los criterios para la revisión de los ítems fueron:

- Valores elevados de desviación típica en las valoraciones.

- Valores V de Akien inferiores a ,7 (equivalentes a puntuaciones medias de la escala inferiores a 3) o cuyo valor crítico a un nivel de confianza de 95\% (límite inferior) es igual o inferior a ,5 (Penfield \& Giacobbi, 2004).

- Diferencias significativas en las valoraciones entre los jueces (p. <,05).

- Observaciones sobre la necesidad de mejorar o eliminar el ítem.

Para el análisis de los ítems aplicamos el índice de Homogeneidad o coeficiente de correlación Pearson estimada mediante "correlación ítem-total corregida" que proporciona el software SPSS. Este coeficiente orienta la selección de ítems para los instrumentos de

Calculado para diez expertos y nueve profesores. Uno de los profesores no aportó valoración cuantitativa del cuestionario. 
medida al indicar hasta qué punto el ítem está midiendo la misma dimensión que el resto de ítems de la dimensión, para lo que se consideran adecuados valores no inferiores a ,20 (Barbero, 2007). Para el caso de cuestionarios multidimensionales Holgado (2010) aconseja estimar este índice única y exclusivamente considerando el conjunto de ítems que se asocian a cada dimensión o concepto para evitar desechar ítems que en su dimensión presenten gran poder discriminativo. Es por ello, que realizamos el análisis de forma independiente a cada uno de los bloques del cuestionario, teniendo presente que no constituían las dimensiones definitivas, las cuales serían estimadas posteriormente mediante Análisis Factorial.

La consistencia interna fue estimada mediante el método alfa de Cronbach que nos proporcionó, además, información sobre el grado en que los ítems afectaban a la consistencia interna de las dimensiones del cuestionario, mediante el cálculo de "alfa de Cronbach si el elemento se ha suprimido".

Los análisis estadísticos estuvieron soportados por los softwares SPSS. El cálculo de $\mathrm{V}$ de Aiken y sus correspondientes intervalos de confianza fueron realizados mediante el programa Visual Basic 6.0 (Merino \& Livia, 2009).

\section{RESULTADOS}

\subsection{VALIDEZ DEL CONTENIDO DEL CUESTIONARIO: LA VISIÓN DE LOS EXPERTOS Y PROFESORES}

\subsubsection{Datos de identificación}

Trece jueces valoraron la sección Datos de identificación del cuestionario (Tabla 5). Los resultados del análisis de frecuencias mostraron una dispersión en las valoraciones aportadas. Un juez experto (Juez 8) y un profesor (Juez 7) valoraron como poco claras, adecuadas y relevantes las preguntas contenidas en este apartado del cuestionario. El resto de jueces indicaron que las preguntas cumplían "Bastante" o "Mucho" con los criterios. Los criterios de Claridad y Adecuación mostraron ponderaciones bajas (menores a ,5) en $\mathrm{V}$ de Aiken para los jueces expertos. Al respecto, estos jueces realizaron valoraciones cualitativas que indicaban sugerencias para la mejora referidas a la modificación de las denominaciones (por ejemplo, Género, Formación recibida, Niveles-años de experiencia) o inclusión de algunas variables sociodemográficas.

Tabla 5. Estadísticos de frecuencia y V de Aiken jueces. Datos de Identificación

\begin{tabular}{|c|c|c|c|c|c|c|c|c|c|c|c|c|c|c|c|}
\hline \multirow{3}{*}{ Criterios } & \multicolumn{10}{|c|}{ Juez Experto } & $A$ & $X$ & $S D$ & $V$ Aiken & $I C$ \\
\hline & \multicolumn{3}{|c|}{ Formación } & \multicolumn{3}{|c|}{ Pruebas } & \multicolumn{4}{|c|}{ Contexto } & & & & & \\
\hline & 1 & 2 & 3 & 4 & 5 & 6 & 7 & 8 & 9 & 10 & & & & & \\
\hline Claridad & -- & 3 & -- & 4 & 4 & 3 & -- & 2 & -- & -- & $4 / 5$ & 3,2 & ,837 & ,733 & ,48* \\
\hline Adecuación & -- & 3 & -- & 4 & 4 & 3 & -- & 2 & -- & -- & $4 / 5$ & 3,2 & 837 & ,733 & , $48^{*}$ \\
\hline Relevancia & -- & 3 & -- & 4 & 4 & 4 & -- & 2 & -- & -- & $4 / 5$ & 3,4 & ,894 & ,8 &, 55 \\
\hline
\end{tabular}




\begin{tabular}{|c|c|c|c|c|c|c|c|c|c|c|c|c|c|c|c|}
\hline \multirow{2}{*}{ Criterios } & \multicolumn{10}{|c|}{ Juez Profesor } & $A$ & $X$ & SD & V Aiken & IC \\
\cline { 2 - 16 } & 1 & 2 & 3 & 4 & 5 & 6 & 7 & 8 & 9 & 10 & & & & & \\
\hline Claridad & -- & 4 & 4 & 3 & 1 & 4 & 1 & 4 & 4 & -- & $6 / 8$ & 3,13 & 1,35 &, 71 &, 52 \\
\hline Adecuación & -- & 4 & 3 & 3 & 3 & 4 & 2 & 4 & 4 & -- & $7 / 8$ & 3,38 &, 744 &, 793 &, 609 \\
\hline Relevancia & -- & 3 & 4 & 4 & 4 & 3 & 3 & 4 & 4 & -- & $8 / 8$ & 3,63 &, 518 &, 876 &, 704 \\
\hline
\end{tabular}

Nota: *= Ponderaciones bajas de V de Aiken; A= Indica acuerdos en valoraciones positivas del total de expertos; IC $=$ Limite inferiores del intervalo de confianza score al 95\%.

Fuente: elaboración propia.

\subsubsection{Valor del conocimiento pedagógico para el ejercicio de la profesión docente}

Entre el 70 y $90 \%$ de los jueces valoraron de forma positiva el contenido de la sección. Las valoraciones medias aportadas para cada una de las dimensiones fueron superiores a 3,00, oscilando entre 3,2 ( $S D=, 789$; Claridad dimensión "Factores influyentes" (C)) hasta 3,9 $(S D=, 316$; Relevancia de la dimensión "Aspectos necesarios para ser profesor" (A)) para jueces expertos.

La magnitud del coeficiente $\mathrm{V}$ de Aiken fue adecuada para todos los grupos de ítems (IC >,5), mostrando valores más bajos para la claridad y relevancia de las dimensiones de esta sección del cuestionario, los cuales fueron ligeramente más elevados para el grupo de profesorado (Figura 2). Al respecto, las pruebas de contraste no indicaron diferencias significativas en las valoraciones de los jueces $(p>, 05)$.

Figura 2. Puntuaciones medias y coeficiente V Aiken dimensiones sección C.1.

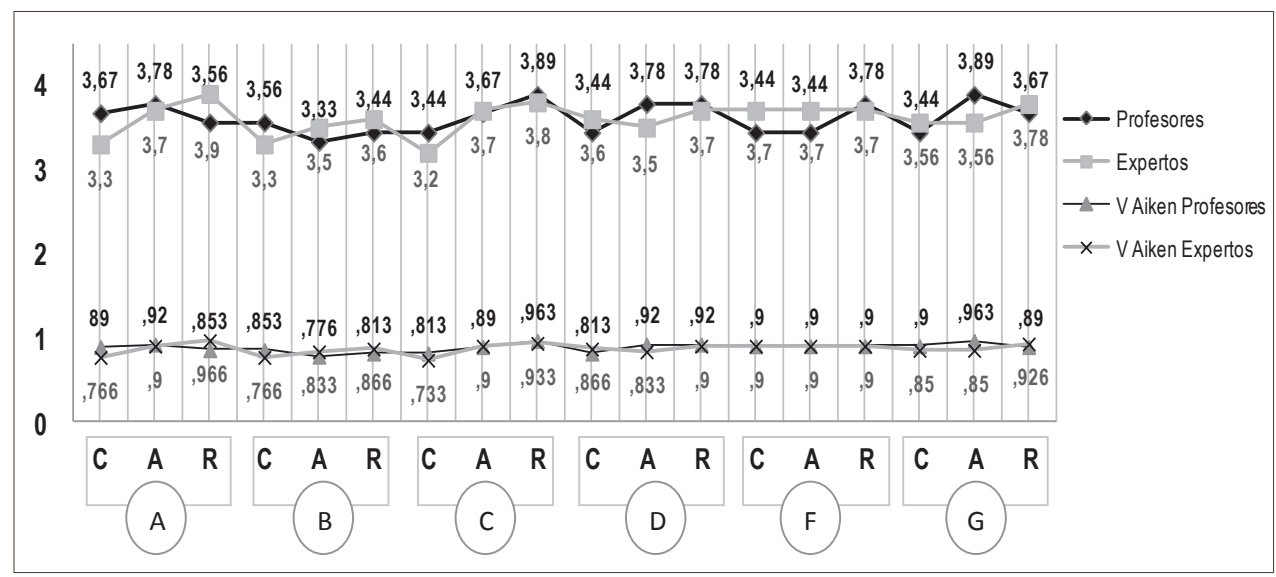

Fuente: elaboración propia 
En concordancia con los indicadores de validez de contenido obtenidos, las valoraciones cualitativas se dirigieron a explicitar la necesidad de mejora de la calidad técnica del contenido de la sección: su redacción, orden de presentación e inclusión de nuevos ítems. Atendiendo a ellas, se realizaron modificaciones en cinco de los ítems pertenecientes a las dimensiones A, B, C y F. Una de las modificaciones más significativas aplicadas a este bloque del cuestionario derivó de las valoraciones cualitativas de los tres jueces expertos especialistas del área de investigación, que indicaron sugerencias referidas al cambio del formato de los ítems -previamente concebidos como preguntas de elección múltiplemediante la inclusión de una escala de valoración tipo Likert de cinco puntos.

\subsubsection{Competencias docentes en el ejercicio de la profesión}

Los valores de los estadísticos de frecuencia muestran entre el 80 y el $100 \%$ de los jueces expertos se ha situado en valores positivos de la escala para valorar el contenido de esta sección. Por otro lado, entre 66,6 y $100 \%$ de los profesores la valoran de forma positiva. Los resultados de validez de contenido mostraron para el criterio claridad los valores más bajos para $\mathrm{V}$ de Aiken $(p \leq, 5)$ y homogeneidad de las respuestas. Especialmente, para las valoraciones aportadas por los jueces expertos sobre la dimensión "Participar en el centro" $(\mathrm{X}=2,90 ; S D=, 994 ; \mathrm{V}$ Aiken=,633); y las aportadas por los profesores a "Innovar" ( $\mathrm{X}=3,00 ; S D=1,00 ; \mathrm{V}$ Aiken=,666). La adecuación y relevancia del conjunto de dimensiones de la sección fueron valorados de forma muy positiva, excepto la adecuación de la dimensión "Participar en el centro" $(\mathrm{X}=3,00 ; S D=, 866 ; \mathrm{V}$ Aiken=,666) (Figura 3). Las pruebas de contraste no indicaron diferencias significativas en las valoraciones de los jueces $(p>, 05)$.

Las valoraciones cualitativas del contenido de esta sección fueron numerosas y tuvieron un peso importante en la mejora y reducción del número de ítems del cuestionario, tal como fue sugerido en la valoración global del cuestionario. Según los jueces expertos en formación docente, algunos de los ítems requerían de una precisión conceptual sobre alguno de sus componentes. Así, verbos como "dominar", "ordenar", fueron sustituidos por "aplicar" y "diseñar", respectivamente. Otras valoraciones estaban referidas a la pertinencia de atender a un solo aspecto en cada ítem, clarificación de las instrucciones para la cumplimentación de la sección, unificación de la terminología empleada, claridad en la redacción, y eliminación de ítems. Atendiendo a las sugerencias aportadas, fueron reformulados, eliminados e integrados un conjunto de ítems, referidos a las dimensiones $\mathrm{V}$ (Participar en el centro) y VI (Actualización e implicación en la profesión).

\subsubsection{Elementos deseables en la Formación Inicial Pedagógica}

Los resultados obtenidos de la valoración del contenido indican valoraciones positivas entre 77,7 y $100 \%$ de los jueces. Los valores medios de las valoraciones para el conjunto de criterios y valoraciones fueron superiores a 3,22 (Figura 4). Cabe destacar que los valores del coeficiente de $\mathrm{V}$ Aiken para las valoraciones de los jueces expertos fueron superiores a ,75 para todos los criterios y dimensiones de la sección. Para el profesorado los valores oscilaron de ,74 (para la claridad de las dimensiones "Procesos metodológicos" (M), "Recursos" (N) y "Evaluación" (P); y la adecuación de "Fórmulas" (K) a ,956 (relevancia de la dimensión "Prácticas docentes" (Q)). Los intervalos de confianza inferiores de V 
Figura 3. Puntuaciones medias y V Aiken jueces sección C.2.

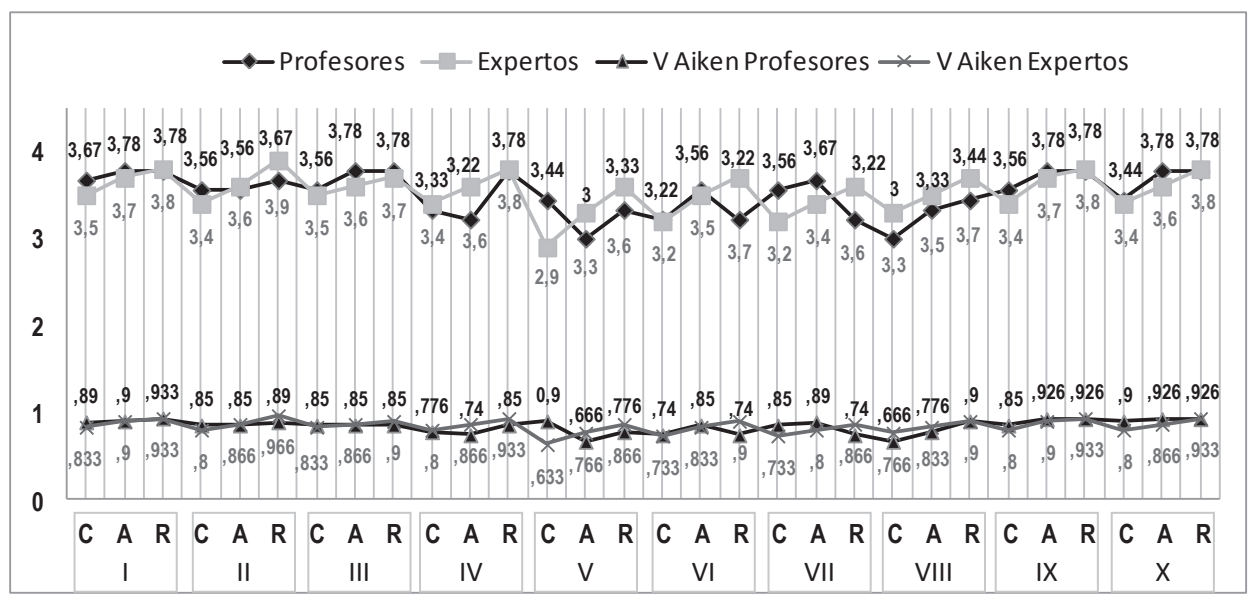

Fuente: elaboración propia

Figura 4. Puntuaciones medias y V Aiken jueces sección C.3.

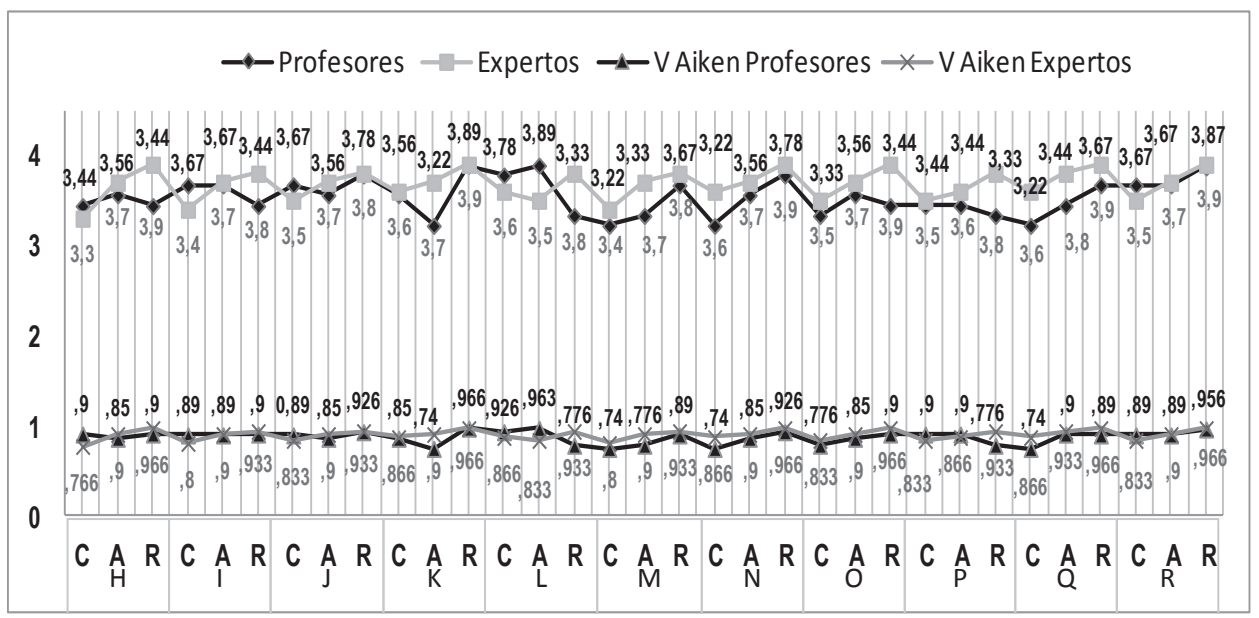

Fuente: elaboración propia

Aiken mostraron valores superiores a ,5. Las pruebas de contraste no indicaron diferencias significativas en las valoraciones de los jueces ( $\mathrm{p}>, 05)$.

Los jueces aportaron sugerencias referidas a la necesidad de clarificar algunos de los enunciados de las dimensiones, mejorar la redacción de algunos de los ítems y ampliar o reducir el universo de ítems de algunas de las dimensiones. Tras el análisis de las valoraciones de los jueces, la sección fue reducida en dos dimensiones ("Fórmulas" y "Recursos") y en un total de 35 ítems y tres preguntas en formato abierto. 


\subsubsection{Valoración global del cuestionario}

Los resultados de los análisis estadísticos muestran valores promedio superiores para las valoraciones de los jueces expertos para casi la totalidad de criterios referidos a la valoración global del cuestionario, destacando los criterios referidos al tiempo de cumplimentación, apariencia y extensión de las instrucciones (Figura 5). Para estos criterios, el valor del estadístico de significación de la prueba U de Mann-Whitney y V Aiken fue inferior a ,5 (Figura 5). Específicamente para el criterio tiempo de cumplimentación los promedios de rango mostraron un valor de 12,55 para los expertos y de Profesores para los profesores 7,17 (sig. $=, 023 ; \chi^{2}=, 814$ ). Para el criterio apariencia del cuestionario el promedio fue de 12,30 para los expertos y 7,44 para los profesores (sig. $=, 047 ; \chi^{2}=3,957$ ). Atendiendo a claridad y extensión de las instrucciones el promedio obtenido para los expertos fue de 12,50 y para profesores de 7,22 (sig. $=, 029 ; \chi^{2}=4,760$ ).

Por otro lado, el contraste de las valoraciones atendiendo al área de especialidad de los jueces expertos indicó diferencias entre los profesores y expertos en investigación y contexto para los criterios tiempo de cumplimentación, apariencia y extensión de las preguntas (expertos en investigación), e instrucciones (expertos contexto).

Figura 5. Puntuaciones medias, V Aiken y U de Mann-Whitney jueces Valoración global

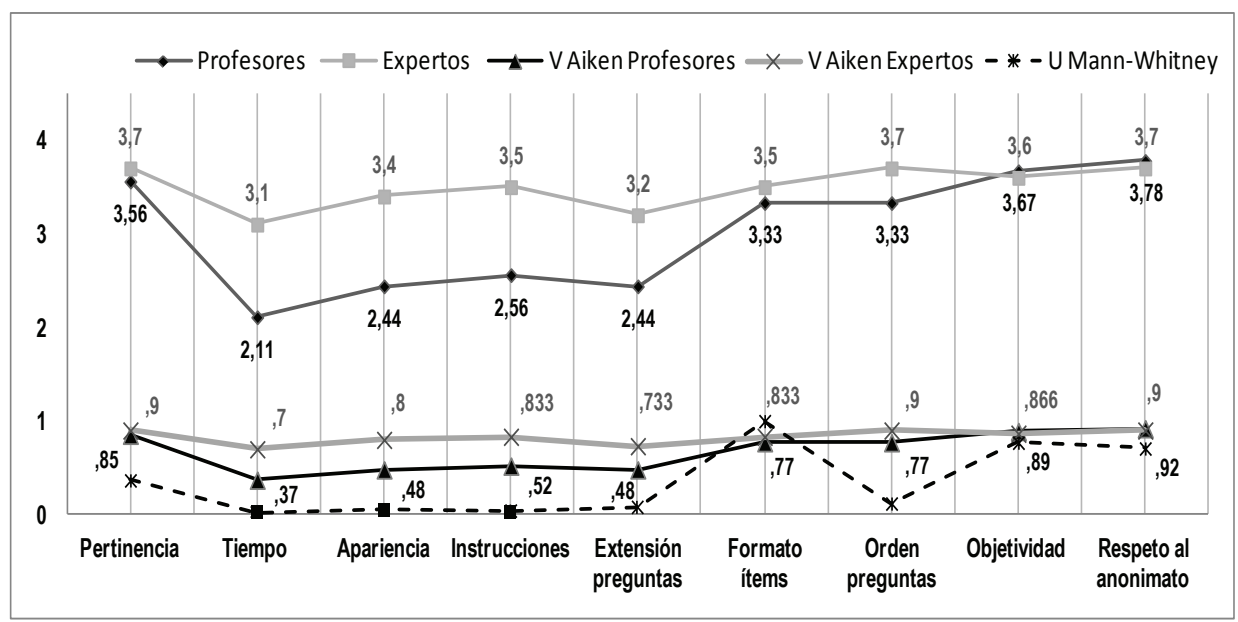

Fuente: elaboración propia

\subsubsection{Valoración de los jueces expertos}

La valoración global realizada por los jueces expertos mostró valores positivos para el conjunto de criterios establecidos (Tabla 6). Los estadísticos de tendencia central y dispersión indicaron valoraciones positivas y homogéneas para los criterios pertinencia del tema, el formato de los ítems, orden de las preguntas, objetividad y respeto al anonimato. La magnitud e intervalo de confianza para $\mathrm{V}$ Aiken mostraron valores adecuados atendiendo a los criterios establecidos $(\mathrm{V} 0=>, 5)$. 
Tabla 6. Valoraciones, V de Aiken y H Kruskal-Wallis. Valoración global del cuestionario por expertos

\begin{tabular}{|c|c|c|c|c|c|c|c|c|c|c|c|c|c|c|c|c|}
\hline \multirow{3}{*}{ Criterios } & \multicolumn{10}{|c|}{ Juez Experto } & \multirow{3}{*}{$A$} & \multirow{3}{*}{$X$} & \multirow{3}{*}{$S D$} & \multirow{3}{*}{$\begin{array}{c}V \\
\text { Aiken }\end{array}$} & \multirow{3}{*}{ IC } & \multirow{3}{*}{$\begin{array}{c}\text { Kruskal- } \\
\text { Wallis }\end{array}$} \\
\hline & \multicolumn{3}{|c|}{ Formación } & \multicolumn{3}{|c|}{ Pruebas } & \multicolumn{4}{|c|}{ Contexto } & & & & & & \\
\hline & 1 & 2 & 3 & 4 & 5 & 6 & 7 & 8 & 9 & 10 & & & & & & \\
\hline $\begin{array}{l}\text { Pertinencia } \\
\text { del tema }\end{array}$ & 4 & 4 & 3 & 4 & 4 & 4 & 4 & 2 & 4 & 4 & 9 & 3,70 & ,675 & ,9 & ,74 & ,619 \\
\hline Tiempo & 3 & 3 & 1 & 4 & 4 & 4 & 3 & 3 & 3 & 3 & 9 & 3,10 & ,876 & ,7 &, 52 &, $020 *$ \\
\hline Apariencia & 3 & 3 & 3 & 4 & 4 & 4 & 4 & 3 & 3 & 3 & 10 & 3,40 &, 516 & ,8 &, 55 &, $045^{*}$ \\
\hline $\begin{array}{l}\text { Claridad y } \\
\text { extensión de } \\
\text { instrucciones }\end{array}$ & 2 & 3 & 4 & 4 & 4 & 3 & 4 & 3 & 4 & 4 & 9 & 3,50 & ,707 & 83 & ,65 & , 420 \\
\hline $\begin{array}{l}\text { Extensión y } \\
\text { cantidad de } \\
\text { preguntas }\end{array}$ & 3 & 3 & 1 & 4 & 4 & 4 & 3 & 3 & 4 & 3 & 9 & 3,20 & ,919 &, 73 &, 55 & ,046* \\
\hline $\begin{array}{l}\text { Formato de } \\
\text { los ítems }\end{array}$ & 3 & 3 & 3 & 4 & 4 & 3 & 4 & 3 & 4 & 4 & 10 & 3,50 & ,527 &, 83 & ,65 & , 142 \\
\hline $\begin{array}{l}\text { Orden de las } \\
\text { preguntas }\end{array}$ & 3 & 4 & 3 & 4 & 4 & 4 & 4 & 3 & 4 & 4 & 10 & 3,70 & ,483 & ,9 & ,74 & ,231 \\
\hline Objetividad & 4 & 3 & 3 & 4 & 4 & 4 & 4 & 3 & 3 & 4 & 10 & 3,60 &, 516 &, 87 &, 7 & ,253 \\
\hline $\begin{array}{l}\text { Respeto al } \\
\text { anonimato }\end{array}$ & 4 & 3 & 3 & 4 & 4 & 4 & 4 & 3 & 4 & 4 & 10 & 3,70 & ,483 & ,9 & ,74 & ,231 \\
\hline
\end{tabular}

Nota: A= Indica acuerdos en valoraciones positivas del total de expertos; $\mathrm{IC}=$ Limite inferiores del intervalo de confianza score al $95 \%$.

Fuente: elaboración propia.

Atendiendo al área de especialización de los jueces expertos, los estadísticos obtenidos en la prueba $H$ Kruskal-Wallis mostraron diferencias $(p<, 05)$ en las valoraciones para los siguientes criterios (Tabla 6): i) tiempo de cumplimentación (sig. $=, 020 ; \chi^{2}=7,833$ ), donde el promedio de rangos fue para Formación docente 3,33, Investigación 9,00 y Contexto 4,50 ; ii) apariencia (sig. $\left.=, 045 ; \chi^{2}=6,188\right)$, con un promedio de rangos para Formación docente 3,50 , Investigación 8,50 y Contexto 4,75; y iii) extensión y cantidad de preguntas donde el promedio fue para Formación docente 3,00, Investigación 8,50 y Contexto 5,13 (sig. $\left.=, 046 ; \chi^{2}=6,175\right)$.

\subsubsection{Valoración del profesorado}

Las valoraciones realizadas por el profesorado se muestran en la siguiente tabla (Tabla 7). Los resultados muestran valores promedio situados en valores positivos y negativos de la escala de valoración, con valores de dispersión elevados para algunos criterios (tiempo de cumplimentación, apariencia, extensión, formato de los ítems). El tiempo de 
Estudios Pedagógicos XLIII, Nº 2: 195-220, 2017

VALOR DEL CONOCIMIENTO PEDAGÓGICO PARA LA DOCENCIA EN EDUCACIÓN SECUNDARIA: DISEÑO Y

VALIDACIÓN DE UN CUESTIONARIO

cumplimentación $(\mathrm{X}=2,11 ; S D=, 928 ; \mathrm{V}$ Aiken=,37) y apariencia $(\mathrm{X}=2,44 ; S D=1,130 ; \mathrm{V}$ Aiken=,48) figuran entre los criterios menos valorados por el profesorado. Cabe destacar que estos criterios presentaron valores significación de $\mathrm{V}$ de Aiken inferiores a ,5, indicando la pertinencia de revisar la extensión y diseño estético del cuestionario. En este sentido, los profesores indicaron un tiempo de cumplimentación medio de una hora, y la pertinencia de diseñar un cuestionario que permita su cumplimentación telemática.

Los criterios que obtienen un valor promedio más elevado y homogéneo por parte de los profesores son la pertinencia del tema $(\mathrm{X}=3,56 ; S D=, 527$; V Aiken=,85), la objetividad de la información que permite recoger el instrumento $(\mathrm{X}=3,67 ; S D=, 500$; V Aiken=,89) y el respeto al anonimato de los encuestados $(\mathrm{X}=3,78 ; S D=, 441 ; \mathrm{V}$ Aiken=,92), mostrando estos criterios valores de significación adecuados para V Aiken. Cabe señalar lo destacado por uno de los jueces participantes sobre la pertinencia de tema investigado: “... creo que son muy pertinentes algunas de las preguntas que haces relacionadas con la formación inicial de los docentes, y te digo también, no sé si lo sabrás, que en este país eso de formación inicial de los docentes "no existe y ni se le ha visto pasar" (Prof. 9).

Tabla 7. Valoraciones y V de Aiken. Valoración global del cuestionario por profesorado

\begin{tabular}{|c|c|c|c|c|c|c|c|c|c|c|c|c|c|c|c|c|}
\hline \multirow{2}{*}{ Criterios } & \multicolumn{10}{|c|}{ Juez Profesor } & \multirow{2}{*}{$A$} & \multirow{2}{*}{$X$} & \multirow{2}{*}{$S D$} & \multirow{2}{*}{$\begin{array}{c}V \\
\text { Aiken }\end{array}$} & \multirow{2}{*}{$I C$} & \multirow{2}{*}{$\begin{array}{l}\text { U Mann- } \\
\text { Whitney }\end{array}$} \\
\hline & 1 & 2 & 3 & 4 & 5 & 6 & 7 & 8 & 9 & 10 & & & & & & \\
\hline $\begin{array}{l}\text { Pertinencia } \\
\text { del tema }\end{array}$ & 4 & 3 & 3 & 4 & 3 & 4 & 4 & 4 & 3 & -- & 9 & 3,56 &, 527 &, 85 &, 67 &, 367 \\
\hline $\begin{array}{l}\text { Tiempo de } \\
\text { cumplimentación }\end{array}$ & 3 & 3 & 1 & 1 & 2 & 2 & 1 & 3 & 3 & -- & $4 / 9$ & 2,11 & ,928 &, 37 &, $21 *$ &, $023 *$ \\
\hline Apariencia & 3 & 4 & 2 & 2 & 4 & 2 & 1 & 3 & 1 & -- & $4 / 9$ & 2,44 & 1,130 & ,48 &, $30 *$ &, $047^{*}$ \\
\hline $\begin{array}{l}\text { Claridad y } \\
\text { extensión de } \\
\text { instrucciones }\end{array}$ & 4 & 3 & 2 & 3 & 3 & 3 & 1 & 3 & 1 & -- & $6 / 9$ & 2,56 & 1,014 &, 52 &, $34 *$ &, $029 *$ \\
\hline $\begin{array}{l}\text { Extensión y } \\
\text { cantidad de } \\
\text { preguntas }\end{array}$ & 4 & 3 & 3 & 1 & 2 & 2 & 3 & 3 & 1 & -- & $5 / 9$ & 2,44 & 1,014 & , 48 &, $30 *$ & ,080 \\
\hline $\begin{array}{l}\text { Formato de } \\
\text { los ítems }\end{array}$ & 4 & 4 & 4 & 4 & 3 & 4 & 3 & 3 & 1 & -- & $8 / 9$ & 3,33 & 1,000 &, 77 &, 59 & 1,000 \\
\hline $\begin{array}{l}\text { Orden de las } \\
\text { preguntas }\end{array}$ & 4 & 3 & 3 & 4 & 4 & 3 & 3 & 3 & 3 & -- & 9 & 3,33 &, 500 &, 77 &, 59 &, 120 \\
\hline Objetividad & 3 & 4 & 4 & 4 & 3 & 4 & 4 & 4 & 3 & -- & 9 & 3,67 &, 500 & ,89 &, 72 &, 770 \\
\hline $\begin{array}{l}\text { Respeto al } \\
\text { anonimato }\end{array}$ & 4 & 4 & 4 & 4 & 3 & 4 & 4 & 4 & 3 & -- & 9 & 3,78 & ,441 & ,92 &, 76 & ,708 \\
\hline
\end{tabular}

Nota: A= Indica acuerdos en valoraciones positivas del total de profesores; $\mathrm{IC}=$ Calculado en base a nueve profesores.

Fuente: elaboración propia. 


\subsection{PRUEBA PILOTO DEL CUESTIONARIO}

A continuación, presentamos los valores obtenidos en la aplicación piloto del cuestionario atendiendo a lo estadísticos empleados, durante la cual no se apreciaron dificultades de comprensión y/o cumplimentación por parte del profesorado participante.

Los valores de consistencia interna aportados por el estadístico alfa de Cronbach (Tabla 8) fueron adecuados para el conjunto de las secciones del cuestionario en la muestra analizada $(\alpha=, 909)$. Atendiendo a cada una de las secciones del cuestionario, el estadístico se muestra adecuado para C.2 $(\alpha=, 933)$ y C.3 $(\alpha=, 888)$.

Tabla 8. Estadísticos de consistencia interna del cuestionario dirigido a profesorado

\begin{tabular}{|l|c|c|c|c|c|}
\hline \multicolumn{1}{|c|}{ Bloque } & $\begin{array}{c}\text { alfa de } \\
\text { Cronbach }\end{array}$ & $\begin{array}{c}\mathrm{N}^{\circ} \text { de } \\
\text { elementos }\end{array}$ & Media & Varianza & $\begin{array}{c}\text { Rango alfa si se } \\
\text { elimina el elemento }\end{array}$ \\
\hline C.1. Valoración del conocimiento &, 678 & 20 & 58,62 & 83,548 & $\begin{array}{c}, 604--->, 699 \\
(\text { ítem C11) }\end{array}$ \\
\hline C.1. Fuentes de adquisición &, 542 & 5 & 16,63 & 7,413 & $\begin{array}{c}\text { Ítem E25 } \\
(\alpha=, 619)\end{array}$ \\
\hline C.2. Competencias &, 933 & 40 & 168,78 & 302,179 &, $929--->, 935$ \\
\hline C.3. Elementos &, 888 & 39 & 164,96 & 175,798 &, $881--->, 893$ \\
\hline Total &, 909 & 104 & 402,94 & 885,809 &, $905--->, 911$ \\
\hline
\end{tabular}

Fuente: elaboración propia.

Para la sección C.1 - calculado para dos ámbitos de contenido diferenciados: valoración del conocimiento pedagógico y valoración de las formas de adquisición del conocimiento-, el estadístico mostró un valor aceptable ( $\alpha=, 678$; Valoración del conocimiento) y bajo ( $\alpha=$ ,542; Fuentes de adquisición), cuyo valores pueden considerarse suficientes cuando se trata de investigación en general (Morales Vallejo, 2007).

Los resultados de Alfa de Cronbach si se elimina el elemento muestran que los coeficientes para cada ítem de las secciones no presentan variaciones elevadas para el contenido global del cuestionario (Tabla 8), excepto para el bloque Valor del conocimiento pedagógico (Tabla 9).

A continuación, mostramos los valores detallados obtenidos del procedimiento de análisis de los ítems atendiendo a los estadísticos de media de escala si el elemento se ha suprimido, varianza de escala si el elemento de ha suprimido, correlación item-total y alfa si se elimina el elemento para el bloque $\mathrm{C} 1$, al presentar resultados significativos para la selección de los ítems del cuestionario. Cabe destacar que para este bloque del cuestionario existían diversos ítems que presentaban valores de homogeneidad menores a 0,20 (Tabla 10), lo que puede estar denotando la existencia de diversas subdimensiones dentro de este bloque del cuestionario. 
Tabla 9. Estadísticos de fiabilidad escala Valor del conocimiento pedagógico. Cuestionario profesorado

\begin{tabular}{|c|c|c|c|c|}
\hline Ítem & $\begin{array}{l}\text { Media de escala } \\
\text { si el elemento se } \\
\text { ha suprimido }\end{array}$ & $\begin{array}{l}\text { Varianza de escala } \\
\text { si el elemento se } \\
\text { ha suprimido }\end{array}$ & $\begin{array}{l}\text { Correlación } \\
\text { ítem-total } \\
\text { corregida }\end{array}$ & $\begin{array}{l}\text { Alfa de Cronbach } \\
\text { si el elemento se } \\
\text { ha suprimido }\end{array}$ \\
\hline $\mathrm{A} 1$ & 56,29 & 64,614 & ,715 & ,604 \\
\hline A2 & 54,14 & 82,429 & ,015 & 686 \\
\hline $\mathrm{A} 3$ & 54,57 & 79,357 & 159 & 675 \\
\hline B4 & 57,00 & 82,200 & ,038 & 683 \\
\hline B5 & 56,14 & 78,229 & , 135 & 681 \\
\hline B6 & 56,43 & 68,257 & 640 & ,621 \\
\hline B7 & 54,57 & 74,757 & ,272 & ,664 \\
\hline B8 & 54,57 & 76,257 & ,278 & 664 \\
\hline B9 & 57,52 & 83,662 &,- 038 & 683 \\
\hline $\mathrm{C} 10$ & 55,67 & 69,233 & ,593 & ,627 \\
\hline $\mathrm{C} 11$ & 56,14 & 84,029 &,- 086 & 699 \\
\hline $\mathrm{C} 12$ & 55,90 & 75,390 & ,190 & ,677 \\
\hline $\mathrm{C} 13$ & 54,76 & 69,790 & ,493 & ,636 \\
\hline $\mathrm{C} 14$ & 55,14 & 76,929 & , 144 & 682 \\
\hline $\mathrm{C} 15$ & 55,24 & 77,190 & ,171 & ,677 \\
\hline $\mathrm{C} 16$ & 55,43 & 68,157 & 670 & 619 \\
\hline $\mathrm{C} 17$ & 57,24 & 81,190 & , 103 & ,678 \\
\hline D18 & 54,14 & 84,129 &,- 083 & 691 \\
\hline D19 & 57,38 & 81,948 & ,134 & 676 \\
\hline D20 & 55,48 & 75,862 & 238 & 668 \\
\hline
\end{tabular}

Fuente: elaboración propia.

Para la dimensión Formas de adquirirlo de la escala Valor del conocimiento pedagógico (Tabla 10) uno de los ítems (E25. Reflexión sistemática sobre la propia práctica docente) mostró valores reducidos en el índice de homogeneidad (correlación total del elemento $=-, 048)$ junto con un aumento considerable en alfa cuando se elimina el elemento. Con la finalidad de explorar de una forma más detallada los valores de homogeneidad para ambas escalas atendimos a la matriz de covarianzas que mostraron valores negativos entre los ítems, especialmente del ítem E25 con el resto de ítems de la dimensión, indicando relaciones inversas entre los ítems (Lind, Marchal \& Wathen, 2008). 
Tabla 10. Estadísticos de fiabilidad para escala

Fuentes de adquisición del conocimiento pedagógico

\begin{tabular}{|c|c|c|c|c|}
\hline Ítem & $\begin{array}{c}\text { Media de escala } \\
\text { si el elemento } \\
\text { se ha suprimido }\end{array}$ & $\begin{array}{c}\text { Varianza de escala } \\
\text { si el elemento se } \\
\text { ha suprimido }\end{array}$ & $\begin{array}{c}\text { Correlación } \\
\text { item-total } \\
\text { corregida }\end{array}$ & $\begin{array}{c}\text { Alfa de Cronbach } \\
\text { si el elemento se } \\
\text { ha suprimido }\end{array}$ \\
\hline E21 & 14,90 & 6,093 &, 182 &, 549 \\
\hline E22 & 12,60 & 4,524 &, 514 &, 349 \\
\hline E23 & 13,50 & 4,466 &, 322 &, 485 \\
\hline E24 & 13,47 & 4,120 &, 544 &, 310 \\
\hline E25 & 12,07 & 7,237 &,- 048 &, 619 \\
\hline
\end{tabular}

Para los bloques "Competencias docentes en el ejercicio de la profesión" (Tabla 10) y "Elementos deseables en la formación inicial pedagógica" (Tabla 8) la aplicación del método de Alfa de Cronbach si se elimina el elemento nos muestra que los coeficientes para cada ítem oscilan entre ,934 y ,929 (Competencias), y entre ,891 y ,881 (Elementos); indicando que ningún ítem presenta grandes variaciones en el contenido total del cuestionario, presentando un índice de homogeneidad adecuado.

\section{CONCLUSIONES}

El trabajo presentado muestra el proceso de construcción y validación de un cuestionario dirigido a conocer el valor concedido por el profesorado al conocimiento pedagógico para la docencia en Educación Secundaria. Con la finalidad de ilustrar dos procesos considerados fundamentales en la construcción de un instrumento de medida antes de proceder a su aplicación definitiva, presentamos un estudio de las evidencias de la validez de contenido y consistencia interna mediante la descripción de dos procedimientos: juicio de expertos y estudio piloto.

La evidencia de validez de contenido fue establecida mediante el procedimiento juicio de diez expertos en diversas áreas de interés para establecer la validez de contenido (investigación, formación docente y contexto en que los profesores de Educación Secundaria desarrollan su acción docente) y diez profesores y profesoras de Educación Secundaria. En nuestro estudio, el procedimiento de selección de los jueces constituyó una tarea esencial para la consecución de unos resultados de calidad (Aiken, 1985; Pedrosa, Suárez-Álvarez \& García-Cueto, 2014). Dicho procedimiento estuvo dirigido a desarrollar una estrategia de triangulación de las valoraciones aportadas por profesionales vinculados al tema de estudio y que ejercen su labor en diversos ámbitos de actuación profesional y experiencia.

En esta lógica de integración de datos, los métodos aplicados ofrecieron las siguientes evidencias de validez de contenido: 
- Consistencia inter-jueces. Los resultados obtenidos mediante la aplicación de procedimientos de análisis de tipo descriptivo y pruebas de contraste de las valoraciones -H de Kruskal-Wallis y U de Mann-Whitney-indicaron valoraciones promedio que se situaban en valores positivos de la escala de valoración, y ausencia de diferencias en las valoraciones de los jueces sobre el conjunto de secciones del cuestionario y criterios de validez de contenido. Consideramos que dichos resultados constituyen una evidencia de validez del juicio de valor aportado por los grupos de jueces.

- Adecuación del contenido establecida mediante coeficiente V de Aiken. Los resultados apoyaron la hipótesis de que la calificación media de los jueces refleja un respaldo positivo de los grupos de ítems. Los valores para el intervalo de confianza (95\% IC inferior) superaron el valor mínimo establecido (,5), excepto para el nivel de claridad y adecuación de dos grupos de ítems del cuestionario, cuyo contenido fue revisado y/o eliminado. Las magnitudes más elevadas se presentaron para la relevancia del contenido alcanzando, en todos los casos, el mínimo más exigente (,75) (Aiken, 1985).

- Valoraciones positivas de la forma del cuestionario. Ambos grupos de jueces valoraron de forma positiva características del cuestionario referidas a la pertinencia del tema, el formato de los ítems, y el orden lógico con el que se presenta, objetividad y respeto al anonimato de los encuestados. Los criterios referidos a su cumplimentación (tiempo, apariencia, extensión de las instrucciones y preguntas) fueron valorados en menor medida por el profesorado, presentando divergencia en las valoraciones de los jueces. Con la finalidad de mejorar dichos aspectos, atendimos a los coeficientes $\mathrm{V}$ Aiken inferiores a ,7 $(\mathrm{IC}=, 5)$ y las valoraciones cualitativas -observaciones- aportadas por los jueces.

La aplicación piloto del cuestionario mostró valores adecuados de consistencia interna global de los bloques del cuestionario. Sin embargo, el estadístico aplicado a cada una de las secciones e ítems mostró valores bajos para la sección "Valor del conocimiento pedagógico para el ejercicio de la profesión docente". Debemos de tomar en consideración que un coeficiente adecuado de fiabilidad y de validez de contenido es una condición necesaria pero no suficiente para afirmar que nuestra escala mide un único rasgo bien definido (Morales Vallejo, Urosa \& Blanco, 2003). Otros aspectos han de ser considerados para conseguir la máxima calidad de una escala como pueden ser los procedimientos más conceptuales dirigidos que pueden modular la selección de los ítems, como es el análisis factorial. No obstante, futuros estudios pueden dirigirse a explorar la estructura factorial del instrumento que se presenta.

\section{REFERENCIAS BIBLIOGRÁFICAS}

Aiken, L.R. (1985). Three Coeficients for Analyzing the Reliability and Validity of Ratings. Educational and Psychological Measurement, 45, 131-142.

Barbero, M.I. (2007). Métodos de elaboración de escalas. Madrid: UNED.

Bolívar, A. (2006). La identidad profesional del profesorado de secundaria: crisis y reconstrucción. Málaga: Archive. 
Bolívar, A., \& Bolívar Ruano, M. (2012). El profesorado de enseñanza media: formación inicial pedagógica e identidad profesional. Ensino Em Re-Vista, 19(1), 19-33.

Bransford, J., Darling-Hammond, L., \& LePage, P. (2005). Introduction. In L. Darling-Hammond \& J. Bransford (Eds.), Preparing teachers for a changing world (pp. 1-39). San Francisco: Jossey Bass.

Burgos, A. (2007). Formación en prevención de riesgos laborales: Bases para la adquisición de una cultura preventiva en los centros educativos (Tesis inédita de doctorado). Universidad de Granada, España.

Cea D’Ancona, M.A. (2001). Metodología cuantitativa. Estrategias y técnicas de investigación social. Madrid: Síntesis.

Cohen, L., Manion, L., \& Morrison, K. (2011). Research Methods in Education. New York, N.Y.: Routledge.

Darling-Hammond, L. (2006). Constructing 21st-Century Teacher Education. Journal of Teacher Education, 57(3), 300-314.

Darling-Hammond, L., \& Baratz-Snowden, J. (2005). A good teacher in every classroom: preparing the highly qualified teachers our children deserve. San Francisco: Jossey-Bass.

Denzin, N.K., \& Lincoln, Y. S. (2005). The Sage Handbook of Qualitative Research (4th ed.). Thousand Oaks: Sage Publications, Inc.

Escobar, J., \& Cuervo, A. (2008). Validez de contenido y juicio de expertos: una aproximación a su utilización. Avances de medición, 6, 27-36.

Escudero, J.M. (2009). La formación del profesorado de Educación Secundaria: contenidos y aprendizajes docentes. Revista de Educación, 350, 79-103.

Esteve, J.M. (2003). La tercera revolución educativa. La educación en la sociedad del conocimiento. Barcelona: Paidós.

Ferrández-Berrueco, R., \& Sánchez-Tarazaga, L. (2014). Competencias docentes en secundaria. Análisis de perfiles de profesorado. RELIEVE, 20(1), 1-20. doi:10.7203/relieve.20.1.3786

González Sanmamed, M. (2009). Una nueva oportunidad para la formación inicial del profesorado de Educación Secundaria. Revista de Educación, 350, 57-78.

Hernández, F. (1998). Para enseñar no basta con saber la asignatura. Barcelona: Paidós.

Hernández, R., Fernández, C., \& Baptista, P. (2014). Metodología de la investigación (6a ed.). México: McGraw-Hill.

Holgado, F. (2010). Análisis de los ítems. En M. I. Barbero, E. Vila \& F.P. Holgado, Psicometría (pp. 407-465). Madrid: Sanz y Torres.

Imbernón, F. (2007). La formación y el desarrollo profesional del profesorado. Barcelona: Graó.

Imbernón, F. (2014). Calidad de la enseñanza y formación del profesorado. Un cambio necesario. Barcelona: Octaedro.

Lind, D. A., Marchal W. G., \& Wathen S. A. (2008). Estadística aplicada a los negocios y la economía. Madrid: Editorial Mc. Graw Hill.

Marcelo, C. (2009). Formalidad e informalidad en el proceso de aprender a enseñar. Revista de Educación, 350, 31-55.

Martín-Romera, A., Molina Ruiz, E., \& Olmo, M. (2015). The value of pedagogical knowledge and initial pedagogical training for secondary school teaching. ECER 2015, Education and Transition. Budapest, Hungría. Recuperado desde http://www.eera-ecer.de/ecer-programmes/ conference/20/contribution/33602/

McMillan, J.H., \& Schumacher, S. (2011). Investigación Educativa. Una introducción conceptual (5a ed.). Madrid: Pearson Educación.

Merino, C., \& Livia, J. (2009). Intervalos de confianza asimétricos para el índice la validez de contenido: Un programa Visual Basic para la V de Aiken. Anales de Psicología, 25(1), 169-171.

Mertens, D. (1998). Research Methods in Education and Psychology. Integrating Diversity with Quantitative and Qualitative Approaches. London: Sage Publications. 
Morales Vallejo, P. (2007). La fiabilidad de los test y escalas. Madrid: Universidad Pontificia de Comillas.

Morales Vallejo, P. (2010). Guía para construir escala de actitudes. Recuperado desde http://www. upcomillas.es/personal/peter/otrosdocumentos/Guiaparaconstruirescalasdeactitudes.pdf.

Morales, P., Urosa, B., \& Blanco, A. (2003). Construcción de escalas de actitudes tipo Likert: una guía práctica. Madrid: La Muralla.

Muijs, D. (2011). Doing Quantiative Research in Educaton with SPSS. Los Angeles: Sage Publications.

Pedrosa, I., Suárez-Álvarez, J., \& García-Cueto, E. (2014). Evidencias sobre la validez de contenido: avances teóricos y métodos para su estimación. Acción Psicológica, 10(2), 3-17.

Penfield, R.D., \& Giacobbi, P. R. (2004). Applying a Score Confidence Interval to Aiken's Item Content-Relevance Index. Measurement in Physical Education and Exercise Science, 8(4), 213225.

Perrenoud, P. (2004). Diez nuevas competencias para enseñar. Invitación al viaje. Barcelona: Graó.

Pontes, A., Ariza, L., \& Del Rey, R. (2010). Identidad profesional docente en aspirantes a profesorado de enseñanza secundaria. Psychology, Society, \& Education, 2(2), 131-142.

Rodríguez, L.J., Alonso, P., Muñiz, L., \& Valcke, M. (2016). ¿Hay un vacío en la formación inicial del profesorado de matemáticas de Secundaria en España respecto a otros países? Revista de Educación, 372, 111-140.

Sánchez-Tarazaga, L. (2012). Importancia de las competencias docentes según el profesorado de secundaria de la provincia de Castellón. Fòrum de Recerca, 17, 403-418.

Sarramona, J. (2012). Desafíos actuales a la profesionalidad de los docentes de secundaria. En E. Tento (Coord.), La escolarización de los adolescentes: desafíos culturales, pedagógicos y de política educativa (pp. 141-162). Buenos Aires: UNESCO.

Shulman, L.S. (2005). Conocimiento y enseñanza: fundamentos de la nueva reforma. Profesorado. Revista de Currículum y Formación del Profesorado, 9(2), 1-30.

Singh, K. (2007). Quantitative Social Research Methods. London: Sage Publications.

Sireci, S., \& Faulkner-Bond, M. (2014). Validity evidence based on test content. Psicothema, 26(1), 100-107.

Tardif, M. (2004). Los saberes del docente y su desarrollo profesional. Madrid: Narcea.

Terigi, F. (Dir.). (2011). Aportes pedagógicos a la reformulación de la formación inicial de los/as profesores/as. Buenos Aires: Ministerio de Educación de la Nación.

Vaillant, D., \& Marcelo, C. (2015). El ABC y D de la Formación Docente. Madrid: Narcea.

Valdés, R., Bolívar, A., \& Moreno, A. (2015). Una valoración de la formación inicial de profesores en España: El Máster en Educación Secundaria. Educação em Revista, 31(3), 251-278. 
Señale con una $X$ o complete donde proceda:

\begin{tabular}{|l|l|l|}
\hline \multicolumn{3}{|c|}{ DATOS DE IDENTIFICACIÓN } \\
\hline Actividad laboral actual: & \multicolumn{2}{|c|}{} \\
\hline $\begin{array}{l}\text { Experiencia: } \\
\text { años }\end{array}$ & Sector profesional: \\
\cline { 2 - 3 } & Inspección Educativa ( ) & Universidad ( ) \\
\hline
\end{tabular}

Valore el nivel de Claridad (C) (los ítems se comprenden fácilmente, es decir, su sintáctica y semántica son adecuadas), Adecuación (A) (los ítems son adecuados para lo que se pretende medir) y Relevancia $(\mathbf{R})$ (los ítems son necesarios o importantes, es decir, deben ser incluidos), de los grupos de ítems del cuestionario utilizando la siguiente escala: $\mathbf{1 =}$ Nada; $\mathbf{2}=$ Poco; $\mathbf{3}=$ Bastante; $\mathbf{4 = M u c h o , ~ y ~ r e c o j a ~ l a s ~ s u g e r e n c i a s ~ q u e ~ c o n s i d e r e ~ o p o r t u n a s ~ e n ~}$ el apartado reservado a "Observaciones".

\begin{tabular}{|l|l|l|l|l|}
\hline \multicolumn{5}{|c|}{ VALORACIÓN DE ÍTEMS DEL CUESTIONARIO } \\
\hline Grupos de ítems & C & A & R & (reflexiones, sugerencias y mejoras): \\
\hline DATOS DE IDENTIFICACIÓN & & & & \\
\hline $\begin{array}{l}\text { VALOR CONOCIMIENTO } \\
\text { PEDAGÓGICO }\end{array}$ & C & A & R & \\
\hline 1. Para ser profesor... & & & & \\
\hline 2. Importancia & & & & \\
\hline 3. Factores influyentes & & & & \\
\hline 4. Mi valoración & & & & \\
\hline 5. Formas de adquisición & & & & \\
\hline 6. Características formación & & & & \\
\hline COMPETENCIAS DOCENTES & C & A & R & \\
\hline Diagnosticar & & & & \\
\hline Planificar & & & & \\
\hline Desarrollar la enseñanza & & & & \\
\hline Orientar & & & & \\
\hline Participar & & & & \\
\hline Actualizarse & & & & \\
\hline Investigar & & & & \\
\hline Innovar & & & & \\
\hline Afrontar deberes éticos & & \\
\hline Evaluar & & & \\
\hline
\end{tabular}


VALOR DEL CONOCIMIENTO PEDAGÓGICO PARA LA DOCENCIA EN EDUCACIÓN SECUNDARIA: DISEÑO Y

VALIDACIÓN DE UN CUESTIONARIO

\begin{tabular}{|l|l|l|l|l|}
\hline ELEMENTOS FORMACIÓN & $\mathbf{C}$ & $\mathbf{A}$ & $\mathbf{R}$ & $\begin{array}{c}\text { Observaciones } \\
\text { (reflexiones, sugerencias y mejoras): }\end{array}$ \\
\hline 8. Principios formativos & & & & \\
\hline 9. Objetivos & & & & \\
\hline 10. Saberes & & & & \\
\hline 11. Fórmulas & & & & \\
\hline 12. Planificación & & & & \\
\hline 13. Procesos metodológicos & & & & \\
\hline 14. Espacios y recursos & & & & \\
\hline 15. Tipos formadores & & & & \\
\hline 16. Requisitos formador & & & & \\
\hline 17. Evaluación & & & & \\
\hline 18. Prácticas docente & & & & \\
\hline
\end{tabular}

A continuación, valore de forma global el cuestionario atendiendo a los aspectos que se exponen a continuación:

$\mathbf{1}=$ Nada $; \mathbf{2}=$ Poco $\mathbf{3}=$ Bastante $\mathbf{4}=$ Mucho

\begin{tabular}{|l|l|l|l|l|l|l|}
\hline \multicolumn{1}{|c|}{ Aspectos } & \multicolumn{3}{c|}{ Valoración } & Observaciones \\
\hline Pertinencia del tema a investigar & 1 & 2 & 3 & 4 & \\
\hline Tiempo de cumplimentación & 1 & 2 & 3 & 4 & \\
\hline $\begin{array}{l}\text { Apariencia: organización y } \\
\text { distribución general }\end{array}$ & 1 & 2 & 3 & 4 & \\
\hline $\begin{array}{l}\text { Claridad y extensión de las } \\
\text { instrucciones }\end{array}$ & 1 & 2 & 3 & 4 & \\
\hline Extensión y cantidad de preguntas & 1 & 2 & 3 & 4 & \\
\hline Formato de los ítems & 1 & 2 & 3 & 4 & \\
\hline Orden de las preguntas & 1 & 2 & 3 & 4 & \\
\hline $\begin{array}{l}\text { Permite recogida de información } \\
\text { objetiva y libre de sesgos }\end{array}$ & 1 & 2 & 3 & 4 & \\
\hline Respeto al anonimato & 1 & 2 & 3 & 4 & \\
\hline
\end{tabular}

